\section{CFAR Data Fusion Center with Inhomogeneous Receivers}

\author{
ANTONI R. ELIAS.FUSTE, Member, IEEE \\ ANTONI BROQUETAS-IBARS, Member, IEEE \\ JORDI PUGA ANTEQUERA, Student, IEEE \\ 1. CARIOS MARIN YUSTE, Student, IEEE \\ Universitat Politènica de Catalunya \\ Spain
}

Detection systems with distributed sensors and data fusion, are increasingly being used by survellance systems. In this work a system formed by $N$ inhomogeneous constant false alarm rate (CFAR) detectors (cell-averaging (CA) and ordered statistic (OS) CFAR detectors) is studied. A recursive formulation is presented of an algorthm that permits for a fixed level of false alarm in the data fusion center, to set the optimum individual threshold levels In the CFAR recelvers and the optimum " $K$ out of $N$ " decision rule in order to maximize the total probability of detection. The algorithm also considers recelvers of different quality or with different communication channel qualities connecting them with the fusion center. This procedure has been applied to several hypothetical networks with distributed CA-CFAR and OS-CFAR receivers and for Rayleigh targets and interference, enabling the inference that in general the fusion decision OR rule is not always the best.

\section{INTRODUCTION}

The most robust surveillance systems that are favored by radar designers today are composed of several spatially distributed sensors and a data fusion center, that combines and controls the information supplied by each sensor [1]. Multistatic radar and/or multiradar systems, present a series of advantages compared with the more classical concentrated systems, for example 1) greater volume of coverage, 2) increase in system reliability and robustness to target fading, and 3) system reconfiguration and thus greater flexibility.

In recent years, several authors have dealt with the subject of distributed detection. Conte, et al. [2], D'Addio, et al. [3], and D'Addio and Farina [4], have studied and classified optimum and suboptimum receivers and fusion centers for different types of targets and interferences with the $O R$ rule as a fusion algorithm and a fusion center with a decision threshold. Other data fusion algorithms based on a fixed threshold in the fusion center were studied by Thomopoulos, et al. [5], for the case in which the decision of each detector is accompanied by further information of its quality. Srinavasan [6] optimized the thresholds at each detector for two fusion algorithms, $\mathrm{OR}$ and $\mathrm{AND}$, considering Rayleigh interference. Finally, Barkat and Varshney $[7,8]$ proposed adaptive threshold techniques in the detectors, to maintain a constant probability of false alarm $\left(P_{\mathrm{fa}_{\mathrm{a}}}\right)$ in the fusion center. The system considered in two cell-averaging constant false alarm rate (CA-CFAR) distributed detectors, assuming Rayleigh interference, and using the fusion rules AND and OR.

Given the nonhomogeneous nature of the interference (clutter clouds), as well as the nonstationary statistics of the interference (multiple targets), the last technique $[7,8]$ turns out to be the most interesting since it allows us to maintain a fixed level of the false alarm in a nonhomogeneous observation scenario.

We propose a similar approach using a rank fusion algorithm. Supposing that we have $N$ adaptive threshold detectors, the data fusion center determines the presence of a target in the tested cell if at least $K$ of the $N$ detectors have made the same decision (rule of rank $K$ with $1 \leq K \leq N$ ). For $N$ receivers this represents all the possible cases between the rule OR $(K=1)$ and the AND rule $(K=N)$.

\section{RANK FUSION RULES FOR DISTRIBUTED DETECTORS}

Fig. 1 presents the problem in consideration, in which a radar observation scenario, that generally includes targets and clutter, is shown. The scenario is observed by $N$ spatially distributed detectors, which in principle can be complete systems ( $N$ radars), or

$0018-9251 / 92 \$ 3.00$ (C) 1992 IEEE 


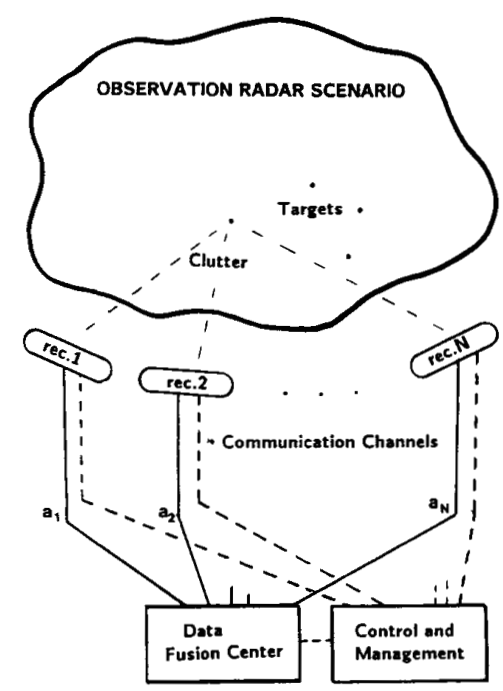

Fig. 1. Distributed detection problem.

$N$ receivers of a multistatic system. The detectors can be different but their characteristics must be perfectly known by the control and management center. Each detector sends its information about the presence or absence of a target in a resolution cell to the Data Fusion Center through a communication channel $\left(a_{i}\right)$. The control and management center determines the detection thresholds (Ti) of each detector, as a function of the data fusion rule and the probability of false alarm $\left(P_{\text {fat }}\right)$ in the Fusion Center, in order to maximize the total probability of detection.

Under the binary hypothesis of detection:

\section{H0: Target signal absent, \\ H1 : Target signal present.}

We can define the probabilities of detection $\left(P \mathrm{~d}_{i}\right)$ and false alarm $\left(P \mathrm{fa}_{i}\right)$ for each detector as

$$
\begin{aligned}
& P \mathrm{~d}_{i}=P(\mathrm{H} 1 / \mathrm{H} 1) \text { in detector " } i \text { ", } \\
& P \mathrm{fa}_{i}=P(\mathrm{H} 1 / \mathrm{H} 0) \text { in detector " } i \text { ". }
\end{aligned}
$$

The detection and false alarm probabilities in the Fusion Center, can be expressed as a function of the probabilities of each detector obtaining the joint probability of $N$ independent events

$$
\begin{aligned}
& P \mathrm{dt}=\sum_{D} R(D) \cdot \prod_{S 0}\left(1-P \mathrm{~d}_{i}\right) \cdot \prod_{S 1} P \mathrm{~d}_{i} \\
& P \text { fat }=\sum_{D} R(D) \cdot \prod_{S 0}\left(1-P \mathrm{fa}_{i}\right) \cdot \prod_{S 1} P \mathrm{fa}_{i}
\end{aligned}
$$

where $\sum$ is the summation of all possible combinations of $D$ decisions, $S 1$ is the group of detectors that has decided the presence of target, $S O$ is the group of detectors that has decided the absence of target, and $R(D)$ is the decision rule.
In general we work with $N$ different receivers, in this way we obtain a decision vector $D$ in the Fusion Center:

$$
D=\left(d_{1}, d_{2}, d_{3}, \ldots, d_{N}\right)
$$

where $d_{1}, d_{2}$, etc. represent the decisions of target presence or absence of each receiver:

$$
\begin{aligned}
& d_{i}=1: \text { Receiver " } i \text { " decides "presence of target" } \\
& d_{i}=0: \text { Receiver " } i \text { " decides "absence of target". }
\end{aligned}
$$

The decision algorithm used as a decision rule $R(D)$ in the Fusion Center is the following rank $K$ rule:

$$
R(D)= \begin{cases}1 \text { (presence of target) } & \text { if } \quad \sum_{i=1}^{N} d_{i} \geq K \\ 0 \text { (absence of target) } & \text { if } \quad \sum_{i=1}^{N} d_{i}<K\end{cases}
$$

For example, with three receivers, the probability of detection $P \mathrm{dt}$ in the Fusion Center is using (1):

$$
\begin{aligned}
P \mathrm{dt}= & R(1,1,1) \cdot P \mathrm{~d}_{1} \cdot P \mathrm{~d}_{2} \cdot P \mathrm{~d}_{3} \\
& +R(1,1,0) \cdot P \mathrm{~d}_{1} \cdot P \mathrm{~d}_{2} \cdot\left(1-P \mathrm{~d}_{3}\right) \\
& +R(1,0,1) \cdot P \mathrm{~d}_{1} \cdot\left(1-P \mathrm{~d}_{2}\right) \cdot P \mathrm{~d}_{3} \\
& +R(0,1,1) \cdot\left(1-P \mathrm{~d}_{1}\right) \cdot P \mathrm{~d}_{2} \cdot P \mathrm{~d}_{3} \\
& +R(1,0,0) \cdot P \mathrm{~d}_{1} \cdot\left(1-P \mathrm{~d}_{2}\right) \cdot\left(1-P \mathrm{~d}_{3}\right) \\
& +R(0,1,0) \cdot\left(1-P \mathrm{~d}_{1}\right) \cdot P \mathrm{~d}_{2} \cdot\left(1-P \mathrm{~d}_{3}\right) \\
& +R(0,0,1) \cdot\left(1-P \mathrm{~d}_{1}\right) \cdot\left(1-P \mathrm{~d}_{2}\right) \cdot P \mathrm{~d}_{3} \\
& +R(0,0,0) \cdot\left(1-P \mathrm{~d}_{1}\right) \cdot\left(1-P \mathrm{~d}_{2}\right) \cdot\left(1-P \mathrm{~d}_{3}\right)
\end{aligned}
$$

if the decision rule is $K=2$, then we have

$$
\begin{aligned}
& R(1,1,1)=R(1,1,0)=R(1,0,1)=R(0,1,1)=1 \\
& R(1,0,0)=R(0,1,0)=R(0,0,1)=R(0,0,0)=0
\end{aligned}
$$

Similar expressions can be obtained for the probability of false alarm $P$ fat in the Fusion Center from (2). The rule can be generalized to the case in which one wants to weigh the decisions of each receiver, since the receivers or the communication channels that connect them to the Fusion Center might have different qualities. For this case we use a vector $A$ in which its components represent the weights of every receiver-communication channel pair according to its performance:

$$
\boldsymbol{A}=\left(a_{1}, a_{2}, a_{3}, \ldots, a_{N}\right)
$$

where $A_{i}$ is the normalized weight factor $\left(0 \leq a_{i} \leq 1\right)$ representative of the quality of the receiver-channel $i$. 
The decision rule $R(D)$, (4), can now be generalized as follows:

$$
R(D)= \begin{cases}1 \text { (target present) } & \text { if } A \cdot D^{T} \geq Z \\ 0 \text { (target absent) } & \text { if } A \cdot D^{T}<Z\end{cases}
$$

where $A \cdot D^{T}$ is the scalar product of the weighting vector $A$ and the decision vector $D$, with $Z$ being a rational number such that $0 \leq Z \leq N$.

The detection and false alarm probabilities in the Fusion Center can be expressed in the unweighted case as follows:

$$
\begin{aligned}
P \mathrm{dt}_{k n} & =\sum_{i=k}^{N}\left\{\left(\sum_{p=0}^{i-k}(-1)^{p} \cdot C(i, p)\right) \cdot\left(\sum_{C_{i N}}\left[\prod_{j} P \mathrm{~d}_{j}\right]\right)\right\} \\
P \mathrm{fat}_{k n} & =\sum_{i=k}^{N}\left\{\left(\sum_{p=0}^{i-k}(-1)^{P} \cdot C(i, p)\right) \cdot\left(\sum_{C_{i N}}\left[\prod_{j} P \mathrm{fa}_{j}\right]\right)\right\}
\end{aligned}
$$

where $P \mathrm{dt}_{k N}$ and $P$ fat $_{k N}$ are the total probabilities of detection and false alarm for a $K$ rank rule applied to $N$ receivers, $P \mathrm{~d}_{j}$ and $P \mathrm{fa}_{j}$ are the probabilities of detection and false alarm for the $j$ th receiver, and $C(i, p)$ are the combinations of $i$ elements taken as groups of $p$ elements:

$$
C(i, p)=\left(\begin{array}{l}
i \\
p
\end{array}\right)=\frac{i !}{p ! \cdot(i-p) !}
$$

and $\sum c_{i N}\left[\prod_{j} P d_{j}\right]$ is the sum of all the possible products of $i$ detection probabilities $\left(P \mathrm{~d}_{j}\right)$ that can be formed from the $N$ receivers.

The same applies for $\sum_{C_{i N}}\left[\prod_{j} P \mathrm{fa}_{j}\right]$. For example:

$$
\begin{aligned}
\sum_{c_{23}}\left[\prod_{j} P \mathrm{~d}_{j}\right]= & P \mathrm{~d}_{1} \cdot P \mathrm{~d}_{2}+P \mathrm{~d}_{1} \cdot P \mathrm{~d}_{3}+P \mathrm{~d}_{2} \cdot P \mathrm{~d}_{3} \\
\sum_{c_{34}}\left[\prod_{j} P \mathrm{~d}_{j}\right]= & P \mathrm{~d}_{1} \cdot P \mathrm{~d}_{2} \cdot P \mathrm{~d}_{3}+P \mathrm{~d}_{1} \cdot P \mathrm{~d}_{2} \cdot P \mathrm{~d}_{4} \\
& +P \mathrm{~d}_{1} \cdot P \mathrm{~d}_{3} \cdot P \mathrm{~d}_{4}+P \mathrm{~d}_{2} \cdot P \mathrm{~d}_{3} \cdot P \mathrm{~d}_{4} .
\end{aligned}
$$

The numerical evaluation of an expression of the form $\sum_{c_{i N}}\left[\Pi_{j} P d_{j}\right]$ using computers is not immediate. However the following recursive formula can be obtained by induction:

$\sum_{c_{i N}}\left[\prod_{j} P \mathrm{~d}_{j}\right]=\sum_{q=0}^{N-i+1} P \mathrm{~d}_{q}\left(\sum_{r=q+1}^{N-i+2} P \mathrm{~d}_{r}\left(\sum_{s=r+1}^{N-i+3} P \mathrm{~d}_{s}(\cdots) \cdot\right)\right)$ in this way, (9) and (10) can be expressed in a compact form that can be directly calculated:

$$
\begin{aligned}
P \mathrm{dt}_{k N}=\sum_{i=k}^{N}\{ & {\left[\sum_{p=0}^{i-k}(-1)^{p}\left(\begin{array}{l}
i \\
p
\end{array}\right)\right] } \\
& {\left[\sum _ { q = 1 } ^ { N - i + 1 } P \mathrm { d } _ { q } \left(\sum_{r=q+1}^{N-i+2} P \mathrm{~d}_{r}\right.\right.} \\
& \left.\left.\left.\cdot\left(\sum_{s=r+1}^{N-i+3} P \mathrm{~d}_{s}(\cdots) \cdot\right)\right)\right]\right\} \\
P \mathrm{fat}_{k N}=\sum_{i=k}^{N}\{[ & {\left[\sum_{p=0}^{i-k}(-1)^{p}\left(\begin{array}{c}
i \\
p
\end{array}\right)\right] } \\
& \cdot\left[\sum _ { q = 1 } ^ { N - i + 1 } P \mathrm { fa } _ { q } \left(\sum_{r=q+1}^{N-i+2} P \mathrm{fa}_{r}\right.\right. \\
& \left.\left.\cdot\left(\sum_{s=r+1}^{N-i+3} P \mathrm{fa}_{s}(\cdots) \cdot\right)\right)\right] .
\end{aligned}
$$

Expressions (15) and (16) represent the Data Fusion Center probabilities of detection and false alarm, processing data from $N$ receivers by applying a $K$ rank fusion rule. That is, the Fusion Center decides the presence of a target if at least $K$ of the $N$ receivers have detected the target.

\section{SYSTEM OPTIMIZATION USING CFAR DETECTORS}

The probability of detection $P \mathrm{~d}_{i}$ and false alarm $P \mathrm{fa}_{i}$ at each receiver can be written as follows [9-11]:

$$
\begin{aligned}
P \mathrm{~d}_{i} & =f(T i, M i, \mathrm{SNR} i) \\
P \mathrm{fa}_{i} & =f(T i, M i)
\end{aligned}
$$

where $T i$ is the scale factor of threshold detection in the CFAR (Fig. 2 and 3), $M i$ is the number of cells of the CFAR, and $\mathrm{SNR}_{i}$ is the signal-to-noise ratio.

Taking into account the expressions (17) and (18) in (15) and (16), it turns out that, in principle, we have three types of optimization.

1) With the $P$ fa $_{i}$ in each receiver fixed, maximize the $P \mathrm{dt}$ of the Data Fusion Center.

2) With the $P$ fat in the Fusion Center fixed, maximize the $P \mathrm{~d}_{i}$ in the receivers.

3) With the $P$ fat in the Fusion Center fixed, maximize also the $P \mathrm{dt}$ in the Fusion Center.

The first optimization is trivial and known, upon fixing the false alarm level at each receiver, the scale factor $T i$ of the threshold level remains fixed. As a consequence, the fusion rule maximizing the probability of detection $P \mathrm{dt}$ in the Fusion Center will be the $O R$ rule $(K=1)$. 


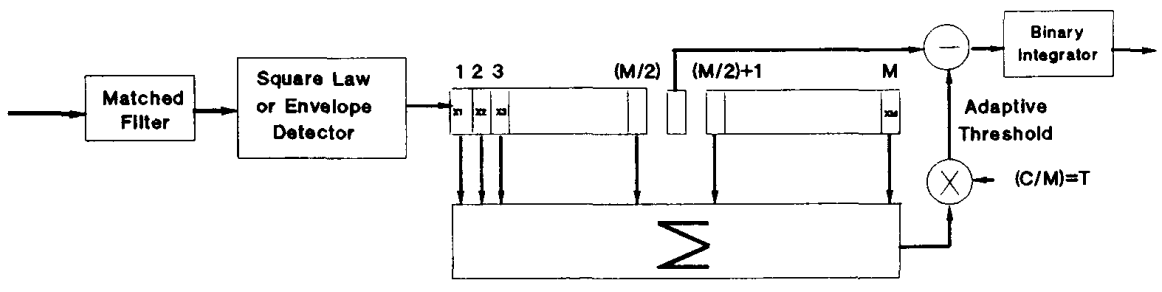

Fig. 2. CA-CFAR schematic.

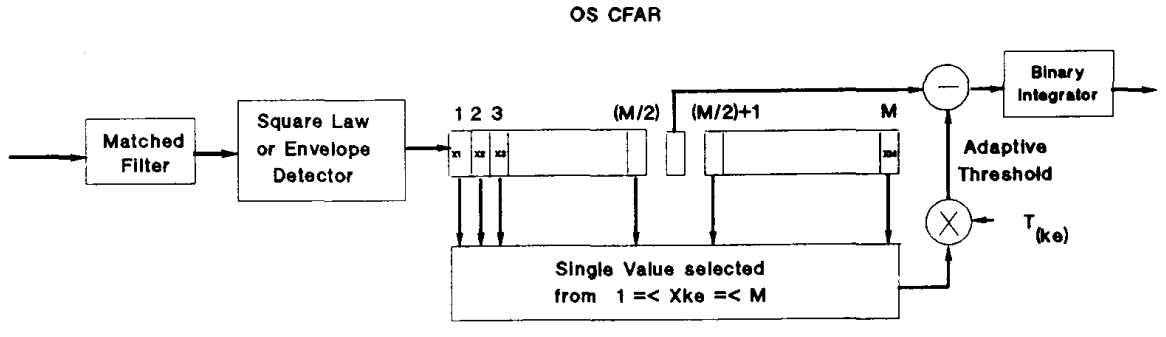

Fig. 3. OS-CFAR schematic.

An attempt to apply the second strategy of optimization, implies the resolution of an underdetermined system of equations. A possible solution would be to maximize the sum of the $P d_{i}$ values, but by whatever means; it turns out to be practically impossible to guarantee the maximum value for each $P \mathrm{~d}_{i}$.

The most interesting optimization is the third one, which has the expected behavior of a CFAR distributed system, where the Data Fusion Center decides the presence or absence of a target. Using this approach, the objective is to optimize the $K$ fusion rule given a network of sensors. For each possible $K$ rule, the proposed algorithm determines the threshold scale factors $T i$ of the receivers, maximizing the total detection probability $P \mathrm{dt}$ in the Fusion Center, given a total constant false alarm probability. The optimum $K$ fusion rule can be chosen by comparing the detection probabilities achieved.

The problem is the same as was solved in [7 and 8] for the AND and OR fusion rules and for two equal and homogeneous CA-CFAR receivers. Generalized here is the method to any set of different and/or inhomogeneous set of receivers (CA-CFAR and OS-CFAR receivers), with different number of estimation cells or different SNR values at each receiver.

We have a function of $N$ variables $P \mathrm{dt}$ :

$$
P \mathrm{dt}=P \mathrm{dt}_{k N}(T 1, T 2, T 3, \ldots, T N)
$$

that we want to maximize under the restriction imposed by another function $P$ fat:

$$
P \text { fat }=P \text { fat }_{k N}(T 1, T 2, T 3, \ldots, T N) .
$$

This is a typical problem of Lagrange multipliers [12]. To solve this, we define the objective function $[7,8]$ :

$$
\begin{aligned}
J(T 1, T 2, \ldots, T N)= & P \mathrm{dt}_{k N}(T 1, T 2, \ldots, T N) \\
& +\beta \cdot\left[P \operatorname{fat}_{k N}(T 1, T 2, \ldots, T N)-\mu\right]
\end{aligned}
$$

where $\beta$ is the Lagrange multiplier, $\mu$ is the desired value for $P$ fat (the probability of false alarm in the Data Fusion Center), and $P \mathrm{dt}_{k N}$ and $P$ fat $_{k N}$ are the probabilities of detection and false alarm in the Data Fusion Center for a fusion rule of $\operatorname{rank} K$ with $N$ distributed receivers. The resulting system of equations is generally nonlinear:

$$
\begin{gathered}
\frac{\partial J(T 1, T 2, \ldots, T N, \beta)}{\partial T 1}=0 \\
\frac{\partial J(T 1, T 2, \ldots, T N, \beta)}{\partial T 2}=0 \\
\vdots \\
\frac{\partial J(T 1, T 2, \ldots, T N, \beta)}{\partial T N}=0 \\
P \operatorname{fat}_{k N}(T 1, T 2, \ldots, T N)=\mu
\end{gathered}
$$

which is a completely determined system with $N+1$ equations and $N+1$ unknowns, corresponding to $T 1, T 2, T 3, \ldots, T N$ and $\beta$. 


\section{DETERMINATION OF OPTIMUM RULE $K$ FOR RAYLEIGH TARGET AND INTERFERENCE}

We assume an environmental interference with a Gaussian probability density function (pdf) for the in-phase and quadrature components, and targets having a similar pdf with a slow fluctuation (Swerling I). After an envelope detection we have a Rayleigh target and a Rayleigh interference, or an exponential target and interference if we detect it with a square detector [9]. In both cases, and with spatially homogeneous interference, the detector that obtains a quasi-optimal constant level of false alarm, or that approaching a Neyman-Pearson detector, is a cell-averaging detector, Fig. 2, [9].

We now consider the problem of obtaining the optimum rank $K$ for data fusion for $N$ different CA-CFAR receivers (with a different number of estimation cells or SNR values for each one). From [9], we know that the probabilities of detection $P d_{i}$ and false alarm $P$ fa $_{i}$ for each detector $i$ are

$$
\begin{aligned}
P \mathrm{~d}_{i} & =\frac{(1+S i)^{M i}}{(1+S i+T i)^{M i}} \\
P \mathrm{fa}_{i} & =\frac{1}{(1+T i)^{M i}} \\
T i & =\frac{C i}{M i}
\end{aligned}
$$

where $S i$ is the SNR at the detector $i, M i$ is the total number of estimation cells of the detector $i, T i$ is the scale factor of the detector $i$, and $C i$ is the $i$ detector threshold value.

The substitution of (23) and (24) in (15) and (16) and using the expressions in (22) gives a system of $N+1$ equations and $N+1$ unknowns that must be solved. For example, for the case of $N=3, K=2$, we have
The system of equations is strongly nonlinear, but fortunately (15) and (16) are recursive simplifying its computer evaluation. We must solve the system for each rank $K$ from $K=1$ to $K=N$ and observe for each $P$ fat which rank $K$ gives the maximum detection probability $P$ dt in the Fusion Center.

If the receivers are ordered statistics OS-CFAR, that have a good behavior in multiple target or inhomogeneous interference situations, the system (22) becomes again nonlinear because the probabilities of detection $P \mathrm{~d}_{i}$ and false alarm $P \mathrm{fa}_{i}$ of each detector are [12]

$$
\begin{aligned}
& P \mathrm{fa}_{i}=k e\left(\begin{array}{l}
M \\
k e
\end{array}\right) \frac{(k e-1) !(T i+M-k e) !}{(T i+M) !} \\
& P \mathrm{~d}_{i}=k e\left(\begin{array}{l}
M \\
k e
\end{array}\right) \frac{(k e-1) !\left[\frac{T i}{(1+S i)}+M-k e\right] !}{\left[\frac{T i}{(1+S i)}+M\right] !}
\end{aligned}
$$

where $k e$ is the order number of the estimating cell taken as representative of the interference level.

To solve the resulting nonlinear systems, the Newton-Raphson iterative method has been used. Applied to the system defined by (22) gives the following matricial expression where the detectors threshold levels $T i$ and $\beta$ are the unknowns:

$$
\begin{aligned}
{[T i, \beta]_{z+1}^{T} } & =[T i, \beta]_{z}^{T}+[\partial F / \partial(T i, \beta)]^{-1} \cdot[F]^{T} \\
{[T i, \beta]_{z} } & =\left[T 1_{z}, T 2_{z}, \ldots, T N_{z}, \beta_{z}\right] \\
{[T i, \beta]_{z+1} } & =\left[T 1_{z+1}, T 2_{z+1}, \ldots, T N_{z+1}, \beta_{z+1}\right]
\end{aligned}
$$

$$
\begin{aligned}
P \mathrm{dt}_{2,3}= & \frac{(1+S 1)^{M 1} \cdot(1+S 2)^{M 2}}{(1+S 1+T 1)^{M 1} \cdot(1+S 2+T 2)^{M 2}}+\frac{(1+S 1)^{M 1} \cdot(1+S 3)^{M 3}}{(1+S 1+T 1)^{M 1} \cdot(1+S 3+T 3)^{M 3}} \\
& +\frac{(1+S 2)^{M 2} \cdot(1+S 3)^{M 3}}{(1+S 2+T 3)^{M 2} \cdot(1+S 3+T 3)^{M 3}}-\frac{2 \cdot(1+S 1)^{M 1} \cdot(1+S 2)^{M 2} \cdot(1+S 3)^{M 3}}{(1+S 1+T 1)^{M 1}(1+S 2+T 2)^{M 2}(1+S 3+T 3)^{M 3}} \\
P \mathrm{fat}_{2,3}= & \frac{1}{(1+T 1)^{M 1} \cdot(1+T 2)^{M 2}}+\frac{1}{(1+T 1)^{M 1} \cdot(1+T 3)^{M 3}}+\frac{1}{(1+T 2)^{M 2} \cdot(1+T 3)^{M 3}} \\
& -\frac{2}{(1+T 1)^{M 1} \cdot(1+T 2)^{M 2} \cdot(1+T 3)^{M 3}} \\
J(T 1, T 2, T 3)= & P \mathrm{~d}_{2,3}(T 1, T 2, T 3)+\beta \cdot\left[P \mathrm{fa}_{2,3}(T 1, T 2, T 3)-\mu\right] .
\end{aligned}
$$


where

$$
\begin{aligned}
{[F]=[} & \frac{J(T 1, T 2, \ldots, \beta)}{\partial T 1}, \frac{\partial J(T 1, T 2, \ldots, \beta)}{\partial T 2}, \ldots, \\
& \left.\times \frac{\partial J(T 1, T 2, \ldots, \beta)}{\partial T N},(P \text { fat }-\mu)\right]
\end{aligned}
$$

and

$$
[\partial F / \partial(T i, \beta)]=\left[\begin{array}{ccccc}
\frac{\partial^{2} J(T 1, T 2, \ldots, \beta)}{\partial T 1^{2}} & \frac{\partial^{2} J(T 1, T 2, \ldots, \beta)}{\partial T 1 \partial T 2} & \ldots & \frac{\partial^{2} J(T 1, T 2, \ldots, \beta)}{\partial T 1 \partial T N} & \frac{\partial^{2} J(T 1, T 2, \ldots, \beta)}{\partial T 1 \partial \beta} \\
\frac{\partial^{2} J(T 1, T 2, \ldots, \beta)}{\partial T 2 \partial T 1} & \frac{\partial^{2} J(T 1, T 2, \ldots, \beta)}{\partial T 2^{2}} & \ldots & \frac{\partial^{2} J(T 1, T 2, \ldots, \beta)}{\partial T 2 \partial T N} & \frac{\partial^{2} J(T 1, T 2, \ldots, \beta)}{\partial T 2 \partial \beta} \\
\ldots & \ldots & \ldots & \ldots & \ldots \\
\ldots & \ldots & \ldots & \ldots & \ldots \\
\frac{\partial^{2} J(T 1, T 2, \ldots, \beta)}{\partial T N \partial T 1} & \frac{\partial^{2} J(T 1, T 2, \ldots, \beta)}{\partial T N \partial T 2} & \ldots & \frac{\partial^{2} J(T 1, T 2, \ldots, \beta)}{\partial T N^{2}} & \frac{\partial^{2} J(T 1, T 2, \ldots, \beta)}{\partial T N \partial \beta} \\
\frac{\partial[P \mathrm{fa} k N(T 1, \ldots, T-\mu]}{\partial T 1} & \ldots & \frac{\partial[P \mathrm{fa} k N(T 1, \ldots, T N)-\mu]}{\partial T N} & \frac{\partial[P \mathrm{fa} k N(T 1, \ldots, T N)-\mu]}{\partial \beta}
\end{array}\right] .
$$

The main drawback of this method is the need of an initial solution near the exact one to converge. To find an initial solution, the bisection method is used; a method that can only be applied to nonlinear equations of only one unknown. Nevertheless, under the assumption that all the receivers are identical, (20) reduces to a function of only one variable:

$$
\begin{aligned}
& \text { if } \quad T 1=T 2=T 3=\cdots=T N=T \\
& \text { then } P \text { fat }_{k N}(T)=\mu .
\end{aligned}
$$

The nonlinear expression (35) is directly solvable by the bisection method, however to initialize the system (29) it is still necessary to find a suitable way to apply the expression (34) and to determine $\beta 0$.

The global solution for the nonlinear system described by the expression (28) is obtained by a computer program with the following main steps.

1) Data are entered specifying the number of receivers $N$, and in the same way the characteristics of each one (Mi, SNR $i$, type of CFAR, etc.) and the probability of false alarm of the network are entered.

2) For the calculation of the initial solution, the program assumes in first place that all the receivers are equal to the first one, and by applying the bisection method, the value of $T 1_{0}$ is found. Secondly, all the receivers are assumed equal to the second one and again the bisection method is applied to find $T 20$. Continuing in this manner, we obtain the initial solution vector $\left[T 1_{0}, T 2_{0}, T 3_{0}, \ldots, T N_{0}\right]$.

3) The determination of $\beta 0$ is done as an average of the different values obtained from each of the equations of system (22) using the different initial thresholds $T i_{0}$ :

$$
\beta_{o}=\frac{1}{N} \sum_{i=1}^{N}-\frac{\frac{\partial P \mathrm{dt}_{k N}(T 1, T 2, \ldots, T N) o}{\partial T i}}{\frac{\partial P \mathrm{fat}_{k N}(T 1, T 2, \ldots, T N) o}{\partial T i}}
$$

4) Once the initial solution vector is completed $\left[T 1_{0}, T 2_{0}, \ldots, T N_{0}, \beta_{0}\right]$, the system (29) is solved iteratively by the Newton-Raphson method for a certain $P$ fat and a fixed fusion rule of rank $K$.

5) The procedure is repeated from step 2 for each fusion rule of rank $K(1 \leq K \leq N)$.

\section{APPLICATION RESULTS}

Several representative situations using networks with CA-CFAR and/or OS-CFAR detectors have been studied according to the proposed method assuming Rayleigh targets and interferences (equations (29)-(33)).

The results for identical receivers (with an equal number of estimation cells) are presented from Figs. 4 and 5 , both showing the probability of detection $P \mathrm{dt}$ in the Fusion Center as a function of the SNR of each receiver (SNR $i$ ) for a system of 7 receivers, Fig. 4 for CA-CFAR receivers, and Fig. 5 for OS-CFAR receivers. For simplicity in the presentation of the results, the same SNR has been used in all the receivers, however the method can be applied to arbitrary situations. The figures show clearly that with the $P$ fat restriction $\left(P\right.$ fat $=10^{-7}$, in the example) the OR rule $(K=1)$ is not always the best one (maximum $P \mathrm{dt})$. For $S N R$ s below $15 \mathrm{~dB}$, the optimal fusion rule is $K=3$.

Similar conclusions can be drawn from Figs. 6 and 7 for the case of five different but homogeneous receivers (CA-CFAR and OS-CFAR, respectively). Also in these cases for SNR below $20 \mathrm{~dB}$ the OR rule isn't the best rule. Fig. 6 (CA-CFAR receivers) shows 


\section{PD-SNR \\ IDENTICAL CA SENSORS \\ $P F A=1 E-7 \quad N=7 \quad M=40$}

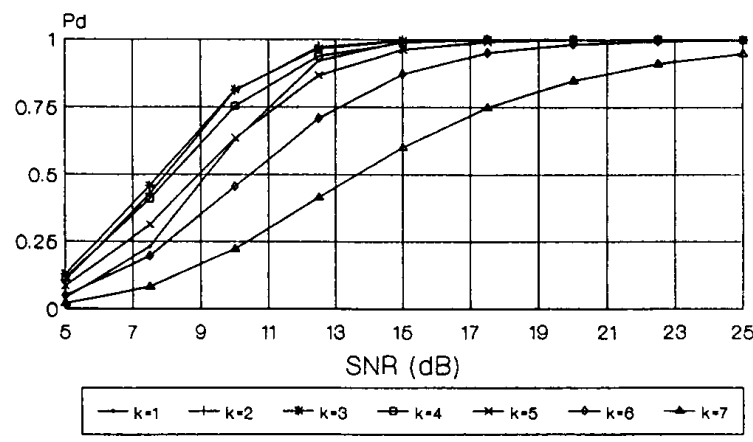

Fig. 4. Performance of seven identical CA-CFAR distributed receivers system for $K=1$ to $K=7$ fusion rules.

\section{PD-SNR}

IDENTICAL OS-CFAR RECEIVERS

$P F A=1 E-7 \quad N=7 \quad M=40 \quad K e=6 / 7 * M=34$

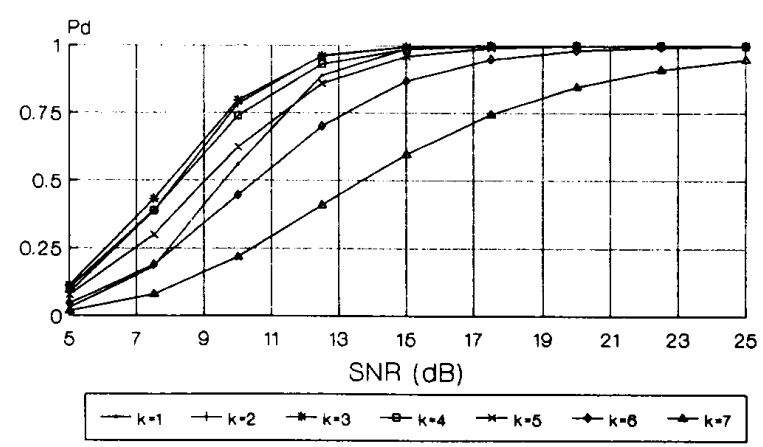

Fig. 5. Performance of seven identical OS-CFAR distributed receivers system for $K=1$ to $K=7$ fusion rules.

that for $P$ fat $=10^{-8}$ and SNR is between 5 to $20 \mathrm{~dB}$ the best rule is $K=2$. For OS-CFAR receivers in this particular case (Fig. 7), $K=2$ is the best one if the SNRis are in the range between 8 to $20 \mathrm{~dB}$, for SNRis lower than $8 \mathrm{~dB}$ and greater than $4 \mathrm{~dB}$ the best one is $K=3$.

Fig. 8 shows a nonhomogeneous distribution with four receivers, using two different CA-CFAR detectors and two different OS-CFAR detectors, with a fixed $P$ fat of $10^{-6}$. The OR rule is the best one for SNR is higher than $18 \mathrm{~dB}$; for SNRis lower than $18 \mathrm{~dB}$ the best rule is $K=2$.

Fig. 9 shows the CFAR loss incurred by using an OS-CFAR instead of a CA-CFAR in homogeneous interference conditions as a function of the decision rule $K$ adopted. The curve represents the additional SNR necessary in an OS-CFAR network of receivers to achieve the same probability of detection $(P \mathrm{dt}=$ 0.5) than in a CA-CFAR network. A horizontal line
$P D-S N R \quad P F A=1 E-8 \quad N=5$ (CA-CFAR) M1-36 M2-24 M3-32 M4-16 M5-34

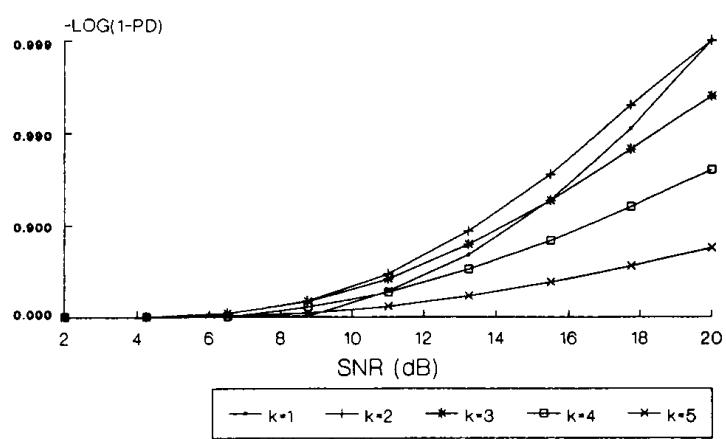

Fig. 6. Performance of five different CA-CFAR distributed receivers system for $K=1$ to $K=5$ fusion rules.

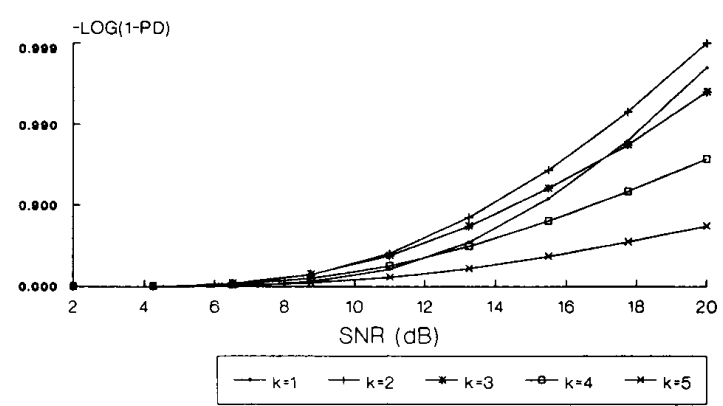

Fig. 7. Performance of five different OS-CFAR distributed receivers system for $K=1$ to $K=5$ fusion rules.

NON-HOMOGENEOUS DISTRIBUTION PD-SNR PFA-1E-6 $\quad \mathrm{N}=4$

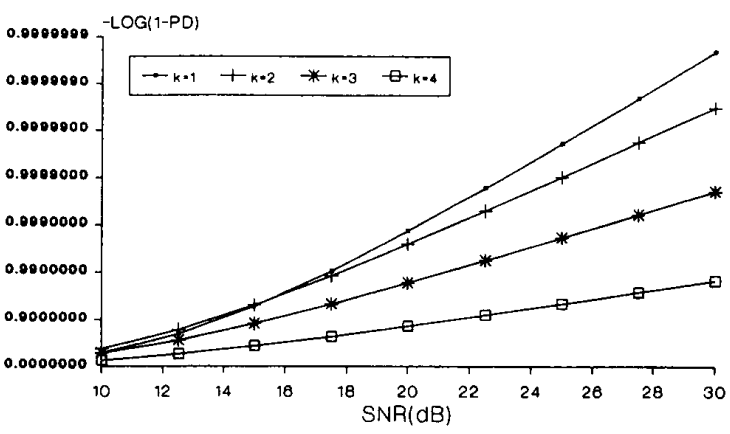

Receivers 1,2 (CA-CFAR) M1-24, M2- 18

Peceivers 3,4 (OS-CFAA) M3-34 Ke3-29, M4-40 Ke4-34

Fig. 8. Performance of four nonhomogeneous parametric distributed receivers system for $K=1$ to $K=4$ fusion rules. 
CFAR LOSS (dB)

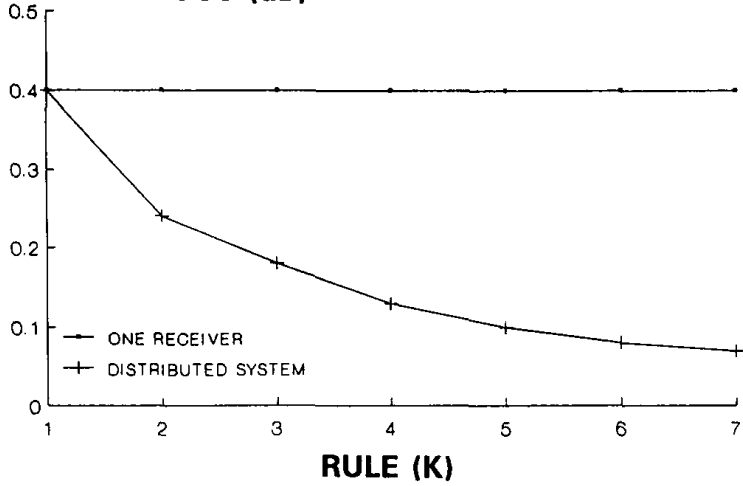

Fig. 9. CFAR loss ( $\triangle$ SNR to achieve $P \mathrm{dt}=0.5$ ) when using OS-CFAR detectors (Fig. 5) instead of CA-CFAR detectors (Fig. 4 ) in homogeneous interference as function of decision rule $K$. Loss corresponding to single detector is shown as a reference.

AVERAGE DETECTION THRESHOLD SNR FOR $P d=0.5 ; P f a=1 E-6$ OS - CFAR USING OPTIMUM RULES FOR MAX PO

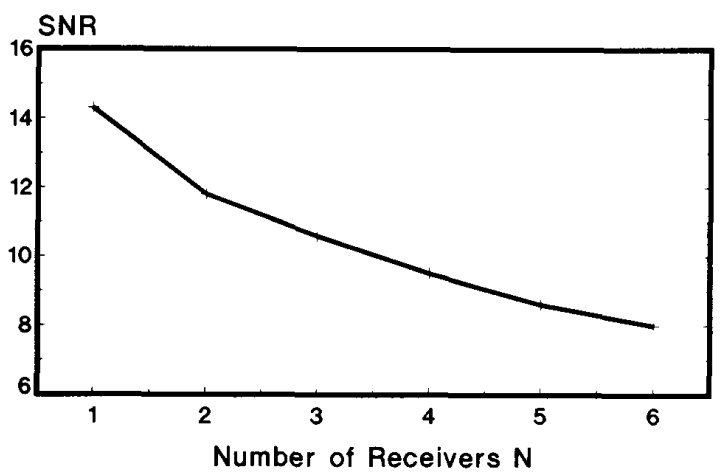

Fig. 10. Average detection threshold (SNR necessary to obtain probability of detection of $P \mathrm{dt}=0.5$ ) as function of number of sensors of network of $N$ OS-CFAR receivers.

represents the loss corresponding to a single receiver case as a reference. It can be observed that as the decision rule $K$ increases, the CFAR loss caused by using OS-CFAR detection instead of CA-CFAR becomes negligible. In more realistic conditions with inhomogeneous interference the OS-CFAR network would keep its intrinsic robustness offering superior performance with respect to the CA-CFAR.

Fig. 10 shows the average detection threshold (SNR necessary to obtain a probability of detection of $P \mathrm{dt}=0.5$ ) as a function of the number of sensors in a network of $N$ OS-CFAR receivers. This result allows the choice of an appropriate compromise between performance and network density (cost). In the case presented, when the number of receivers exceeds 5 or 6 , the addition of a new receiver does not significantly improve the detection performance of the network.

\section{CONCLUSIONS}

A recursive algorithm has been formulated that permits the study of the rank fusion rules in a Data Fusion Center managing a distributed network with $N$ CFAR receivers or detectors. The algorithm obtains the threshold levels of the receivers in order to maximize the total probability of detection keeping the total false alarm constant. The network can consist in an arbitrary combination of CA-CFAR or OS-CFAR detectors with different characteristics and working in different conditions. For a given network the evaluation of the possible rank fusion rules shows the optimum choice offering the highest probability of detection.

The algorithm has been applied to hypothetical networks with CA-CFAR and OS-CFAR detectors and for Rayleigh targets and interference. The following remarks can be made.

1) Upon fixing the probability of false alarm in the Data Fusion Center ( $P$ fat), the OR fusion rule isn't always the rule which maximizes the total detection probability $(P \mathrm{dt})$ in the Fusion Center. Regardless of whether the detectors are identical or not, once the $P$ fat has been fixed, the algorithm allows to find the optimum rank fusion rule $K$, with $1 \leq K \leq N$ that maximizes $P$ dt.

2) The OR fusion rule $(K=1)$ maximizes the $P$ dt (becomes optimal), when the $P$ fat is low and the quality of the receivers is high, either by using a large number of estimation cells $M i$, or by increasing the number of receivers $N$, or in the case of working with high SNRs.

3) The requirement of low values of $P$ fat implies an increment in the threshold factors $T$ and/or in an increase of the rank $K$ of the optimum decision rule.

4) As expected, the $P$ dt increases with the number of receivers as well as their quality for all the possible decision rules $K$. For systems with different receivers, the threshold scale values $T i$ increase as the quality of the detectors decreases.

5) For a given block of detectors, optimizing the network under the restriction of constant total false alarm probability ( $P$ fat) produces a higher $P \mathrm{dt}$ in the Fusion Center than optimizing the network based on keeping a constant false alarm of each receiver independently.

6) The simulations show that although CFAR loss decreases with the number of fused receivers $N$, the improvement is not significative when $N$ is greater than 6 or 7 .

7) Using OS-CFAR receivers the global CFAR loss is comparable to loss of a CA-CFAR network, but it keeps the robustness in conditions of strong inhomogeneous environment (multiple targets and clutter edges). 


\section{REFERENCES}

[1] Farina, A., and Galati, G. (1988)

Curso de Procesado de Señal y Datos Radar. C.E.M. Madrid, Apr. 25-29, 1988.

(2) Conte, E., D'Addio, E., Farina, A., and Longo, M. (1983) Multistatic radar detection: Synthesis and comparison of optimum and sub-optimum receivers.

IEE Proceedings, 130, Pt. F (Oct. 1983), 484-494.

[3] D'Addio, E., Farina, A., Conte, E., and Longo, M. (1985) Multistatic detection of radar signals with Swerling fluctuation type model. Rivista Tecrica Selenia, 9, 4 (1985), 11-17.

[4] D'Addio, E., and Farina, A. (1986)

Overview of detection theory in multiestatic radar. IEE Proceedings, 133, Pt. F, 7 (Dec. 1986), 613-623.

[5] Thomopoulos, S. C. A., Viswanathan, R., and Bougoulias, D. (1987)

Optimal decision fusion in multiple sensor systems. IEEE Transactions on Aerospace and Electronic Systems, AES-23, 5 (Sept. 1987), 644-653.

[6] Srinavasan, R. (1986)

Distributed radar detection theory.

IEE Proceedings, 133, Pt. F, 1 (Feb. 1986), 55-60.

[7] Barkat, M., and Varshney, P. K. (1987)

Cell-averaging CFAR detection with distributed radars and data fusion.

In Proceedings of the International Conference on Radars, London, Oct. 19-21, 1987, 55-60.
[8] Barkat, M., and Varshney, P. K. (1989)

Decentralized CFAR signal detection.

IEEE Transactions on Aerospace and Electronic Systems, 25, 2 (Mar. 1989), 141-149.

[9] Finn, H. M., and Johnson, R. S. (1968) Adaptative detection mode with threshold control as a function of spatially sampled clutter level estimates. RCA Review, 29, 3 (Sept. 1968), 414-464.

[10] Rickard, J. T., and Dillard, G. M. (1982) Adaptative detection algorithms for multiple target situations. IEEE Transactions on Aeraspace and Electronic Systems, AES-18, 1 (Jan. 1982), 102-113.

[11] Rohling, H. (1983)

Radar CFAR thresholding in clutter and multiple target situations.

IEEE Transactions on Aerospace and Electronic Systems, AES-19, 4 (July 1983), 608-621.

[12] Arbenz, K., and Wohlhauser, A. (1986) Advanced Mathematics for Procticing Engineers. Dedham, MA: Artech House, 1986. 

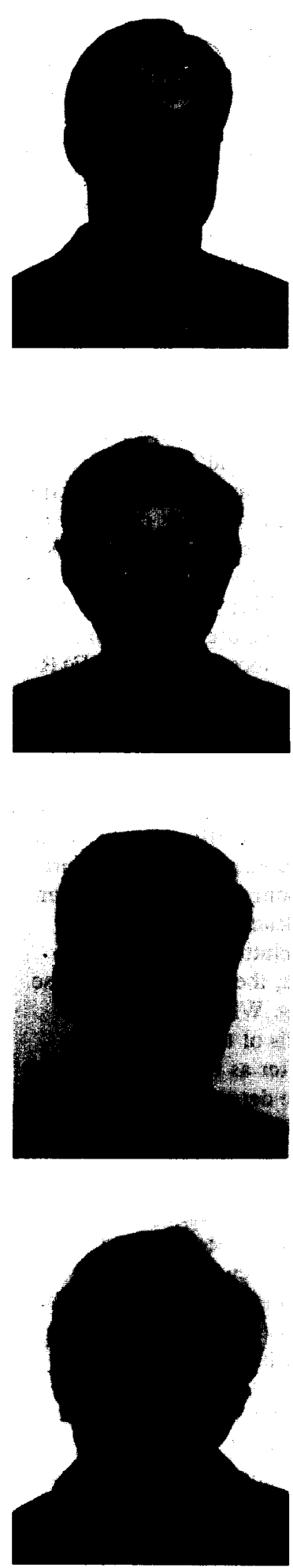

Antoni R. Elias-Fusté (S'78-M'82) was born in Cervera, Spain, in 1954. He received the Ingeniero and Doctor Ingeniero degrees in telecommunication engineering, both from the Universitat Politècnica de Catalunya (U.P.C.) in 1978 and 1982 , respectively.

In 1976 he joined the Antenas-Microondas-Radar Group of the Department of Signal Theory and Communications of the Escuela Técnica Superior de Ingenieros de Telecomunicación de Barcelona, and he works in microwave characterization and RF systems design. During the period 1983-1984 he had a Research French Government Grant at the Centre National des Etudes des Telecommunications (CNET) Lannion (France), working on microwave nonlinear systems. He became Associate Professor in 1985 and Professor in 1990 at the U.P.C., engaged in research in the field of radar.

Dr. Elias-Fuste is an IEEE and AOC member. Currently, he is the Chairman of the AES Spanish Chapter. He is author and coauthor of several reports for the industry and papers published in technical journals and conference proceedings.

Antoni Broquetas-Ibars (S'84-M'90) was born in Barcelona, Spain, in 1959 He received the Ingeniero degree in telecommunication engineering from the Universitat Politècnica de Catalunya (U.P.C.) in 1985, and the Doctor Ingeniero degree in Telecommunication Engineering for his work in microwave tomography in 1989.

In 1984 he joined the Electrophysics group of the U.P.C. working in microwave systems and digital radio links. During 1986 he was a research assistant in the Portsmouth Polytechnic (U.K.) involved in propagation studies. Currently he is Associate Professor of the U.P.C. where he is engaged in research in the fields of microwave imaging and radar.

Jordi Puga Antequera ( $\mathrm{S}^{\prime} 89$ ) was born in 1965 . He received the Ingeniero degree in telecommunication engineering from the Universitat Politecnica de Catalunya (U.P.C.) in 1989. Since 1988 he has been teacher of the Spanish "Universidad Nacional a Distancia" in the fields of Computer Science and Physics. In 1990 he joined the Communications and Technical Systems section of SEMA-GROUP Barcelona, where he is involved in optimization study of data line costs for the S.G.O. (Sistema de Gestión de Ordenes) and development of a communication link using X25 lines of the Stock Exchange of Barcelona, and PRODAT-2 project, instigated by the European Space Agency (ESA), a data communication system, which, by means of a satellite link, provides mobiles units with a messaging service.

Juan C. Marin Yuste (S'90) was born in Barcelona, Spain, in 1965. He received the Ingeniero degree in telecommunication engineering in 1989 from the Universitat Politecnica de Catalunya. In 1990 he joined the Antenna-Microwave-Radar group of the Signal Theory and Communications Department, working in radar systems. 